\title{
A disintegrin and metalloprotease 10-containing exosomes derived from nasal polyps promote angiogenesis and vascular permeability
}

\author{
WEI ZHANG $^{1 *}$, JIE ZHANG $^{1 *}$, LEI CHENG ${ }^{2}$, HAOSHENG NI $^{1}$, BO YOU ${ }^{1}$, YING SHAN $^{1}$, \\ LILI BAO ${ }^{1}$, DI WU ${ }^{1}$, TING ZHANG ${ }^{1}$, HUIJUN YUE ${ }^{1}$ and JING CHEN ${ }^{1}$ \\ ${ }^{1}$ Department of Otorhinolaryngology, Affiliated Hospital of Nantong University, Nantong, Jiangsu 226001; \\ ${ }^{2}$ Department of Otorhinolaryngology, The First Affiliated Hospital of Nanjing Medical University, \\ Nanjing, Jiangsu 210029, P.R. China
}

Received July 2, 2017; Accepted February 9, 2018

DOI: $10.3892 / \mathrm{mmr} .2018 .8634$

\begin{abstract}
Abnormal angiogenesis and vascular permeability is important for the formation of nasal polyps (NPs). Increasing evidence has indicated that exosomes serve a vital role in modulating angiogenesis and vascular permeability. A disintegrin and metalloprotease 10 (ADAM10), an important type of proteinase that is overexpressed in various diseases, can influence angiogenesis and vascular permeability and has been observed in healthy nasal exosomes. To the best of our knowledge, the expression levels and the function of ADAM10 in NLF-derived exosomes from NPs has not been demonstrated previously. In order to determine the influence of exosomes derived from nasal lavage fluid (NLF) on angiogenesis and vascular permeability, 25 nasal polyp patients and 15 healthy volunteers were enrolled in the present study. NLF was collected from all of the subjects. Exosomes were isolated from NLF, visualized under transmission electron microscope and identified using western blot analysis. The effect of exosomes on human umbilical vein endothelial cells (HUVECs) was measured by tube formation and permeability assays in vitro. The expression of exosomal ADAM10 was also analyzed by western blotting. NLF-derived exosomes from NPs influenced proliferation, tube formation and the permeability of HUVECs. ADAM10 was highly expressed in NLF-derived exosomes from NPs when compared with healthy volunteers. Thus, NLF-derived exosomes from NPs promoted angiogenesis and vascular permeability, which may be associated with abundant ADAM10 in NP exosomes.
\end{abstract}

Correspondence to: Dr Jing Chen or Dr Huijun Yue, Department of Otorhinolaryngology, Affiliated Hospital of Nantong University, 20 Xisi Road, Nantong, Jiangsu 226001, P.R. China

E-mail: chenjing0408@hotmail.com

E-mail: yhjent@163.com

*Contributed equally

Key words: exosomes, A disintegrin and metalloprotease 10, nasal polyps, angiogenesis, vascular permeability

\section{Introduction}

Chronic rhinosinusitis with nasal polyps (CRSwNP) is a major health care problem that affects about 0.5 to $4 \%$ of the general population (1). CRSwNP leads to high management costs and poor quality of life (2). Histomorphological transformation in nasal polyps (NPs) involves infiltration of inflammatory cells, abnormal angiogenesis, remarkable edema, submucosal fibrosis, a decreased number of mucous glands and mucosal epithelial hyperplasia (3). Many researches indicate that the increase in capillary and basilar membrane permeability in NPs leading to severe edema and polyp growth $(4,5)$. However, the exact pathogenic mechanisms underlying NPs are still largely unknown. Therefore, further research of capillary may help to illuminate the pathogenesis of NPs.

Exosomes, as an important communication tool among various cells, are very popular in present clinical research $(6,7)$. Exosomes are small, 50-100 nm membrane vesicles, which are released extracellularly after fusion of multivesicular endosomes with the cell membrane (8). Exosomes exist in various body fluids such as serum, urine, and breast milk (9-12). Recently, some researches have shown that exosomes in human nasal lavage fluid (NLF) can be as a new diagnostic indicator of upper respiratory tract disease (13-15). Exosomes containing abundant protein, mRNA, miRNA and some other bioactive substances are involved in immunoregulation, extracellular matrix remodeling, cellular signal transduction and so on $(16,17)$. Lee et al (18) have found that in hypoxia condition, exosomes can promote pathological angiogenesis in a zebrafish tumor model. Kalani et al (19) also have shown that exosomes can regulate permeability of human umbilical vein endothelial cells (HUVECs). Although there have been many reports about exosomes, the role of exosomes in NPs are still unknown.

A Disintegrin And Metalloproteases (ADAMs) are family of proteases that are responsible for the liberation of a variety of cell surface expressed proteins. Recently, some studies have identified that ADAM10 can influence angiogenesis (20) and vascular permeability (21), which then inducing the genesis and progression of some inflammatory diseases and cancers. In turn, ADAM10 may be a potential therapeutic target in these diseases $(22,23)$. Particularly, ADAM10 
has been identified as a novel binding partner of VEGFR2 which influencing the role of VEGF-VEGFR in vascular permeability, and ADAM10 regulates endothelial permeability by proteolysis of vascular endothelial cadherin in the progressing of atherosclerosis (24-26). Recently, ADAM10, but not other metalloprotionases, has been identified in healthy nasal exosomes in Lässer et al 2016 Journal of Translational Medicine (15). However, the expression level and the function of ADAM10 in NLF-derived exosomes from NPs has not been demonstrated. In this study, exosomes were successfully isolated from NLF, and we proved that the NLF-derived exosomes from NPs promoted angiogenesis and vascular permeability. Besides, we revealed that ADAM10 was highly expressed in NLF-derived exosomes from NPs. Therefore, we speculated that ADAM10 may play an important role in the effects of NLF-derived exosomes from NPs on angiogenesis and vascular permeability.

\section{Materials and methods}

Clinical specimens and cell lines. A total of 25 patients with CRSwNP were enrolled in this prospective clinical study from the Affiliated Hospital of Nantong University (Nantong, China). The diagnosis of CRSwNP was made to the criteria established by the European Position Paper on rhinosinusitis and nasal polyps guidelines (1). Each patient underwent routine workup before operation, including a medical interview, a physical examination, anterior rhinoscopy, nasal endoscopy, a computerized tomography (CT) scan, blood tests, and skin prick tests (SPT). No patient had a history of allergic rhinitis, asthma, or aspirin sensitivity, and none had received systemic and/or topical nasal steroids treatment at least 3 weeks before surgery. A total of 15 healthy volunteers were recruited to collect their NLF.

HUVECs (ScienCell Research Laboratories, Inc., San Diego, CA, USA) were cultured in DMEM low glucose (HyClone, Logan, UT, USA) and incubated at $37^{\circ} \mathrm{C}$ containing $5 \% \mathrm{CO}_{2}$. Written informed consent was obtained from all the NPs patients and healthy volunteers, and the study was approved by the Medical Ethics Committee of the Affiliated Hospital of Nantong University.

NLF collection. NLF was collected from NP patients and healthy volunteers respectively. We used an established method with minor adjustments (27). In brief, $5 \mathrm{ml}$ of $0.9 \%$ saline was instilled in right nostril of the person while the head leaned back at an angle about $30^{\circ}$ and the soft palate closed. The NLF was obtained by bending the head forward and passively collecting the fluid. And then the procedure was repeated in the left nostril. The NLF we collected was then passed through a cell strainer $(100 \mu \mathrm{m})$ and promptly centrifuged $(3,000 \mathrm{x} \mathrm{g}$, $20 \mathrm{~min}, 4^{\circ} \mathrm{C}$ ) to remove crust and debris. The supernatant of $\mathrm{NLF}$ was stored at $-80^{\circ} \mathrm{C}$ before exosomes isolation.

Exosomes isolation and purification. Exosomes were isolated from NLF of NP patients (NPs exosomes) and healthy volunteers (normal exosomes) using a previously described method $(28,29)$ with the modifications that included differential centrifugation of NLF $\left(6,000 \mathrm{x} \mathrm{g}\right.$ for $30 \mathrm{~min}$ at $4^{\circ} \mathrm{C}$ and $10,000 \times \mathrm{g}$ for $60 \mathrm{~min}$ at $\left.4^{\circ} \mathrm{C}\right)$ followed by ultrafiltration $(0.2-\mu \mathrm{m}$ filter; Sarstedt, Nümbrecht-Rommelsdorf, Germany) and $\mathrm{qEV}$ size-exclusion columns (iZON Science, Christchurch, New Zealand). The supernatant of NLF was then ultracentrifuged $(100,000 \mathrm{x} \mathrm{g})$ for $60 \mathrm{~min}$ at $4^{\circ} \mathrm{C}$ (Type $90 \mathrm{Ti}$ Rotor; Beckman Coulter, Inc., Brea, CA, USA) to pellet the exosomes. The exosome pellets were then washed once with PBS.

Electron microscopy. The exosome pellets were fixed with $2.5 \%$ glutaraldehyde and centrifuged at $100,000 \mathrm{x}$ g to remove the glutaraldehyde. Afterwards, the pellets were stained by $3 \%$ aqueous phosphotungstic acid and fixed on copper mesh formvar grids. Samples were examined in a JEOL Transmission Electron Microscope (JEM-1230; JEOL, Ltd., Tokyo, Japan).

Nanoparticle tracking analysis (NTA). NTA utilizes the properties of both light scattering and Brownian motion in order to obtain the particle size distribution of samples in liquid suspension.The NanoSight range of instruments provides high resolution particle size, concentration and aggregation measurements. Appropriate exosomes concentration were used to assess the size distribution by Zeta View (Particle Metrix GmbH, Meerbusch, Germany).

In vitro tube formation assay. Matrigel tube formation assays using growth factor-reduced Matrigel (BD Biosciences, Franklin Lakes, NJ, USA). HUVECs were seeded at $1.5 \times 10^{4}$ cells/well of 96 -well plates precoated with Matrigel and cocultured with exosomes for $6 \mathrm{~h}$ at $37^{\circ} \mathrm{C}$. Images of the tube formation were obtained using an inverted microscope. Tubes were defined as elongated connecting branches between 2 identifiable HUVECs.

In vitro permeability assay. HUVECs were seeded on transwell chamber $(0.4-\mu \mathrm{m}$ pore size; Costar, Corning, NY, USA) in 24-well plates and grown until reaching confluence. Fluorescein isothiocyanate (FITC)-dextran $(1 \mathrm{mg} / \mathrm{ml})(M$, 40 000; Thermo Fisher Scientific, Inc., Waltham, MA, USA) was then added to the upper chamber. At the indicated time points 50- $\mu$ l samples were taken from the lower chamber and replaced with the same volume of culture medium. A fluorescence plate reader (Lambda Fluoro 320; MWG Biotech, Ebersberg, Germany) was using to measure the fluorescent content of samples.

Cell viability assay. Cells were seeded into 96-well plates and assessed by CCK-8 assay (Beyotime Institute of Biotechnology, Haimen, China). The absorbance of each well was read on a microplate reader (F-2500 Fluorescence Spectrophotometer; Hitachi, Ltd., Tokyo, Japan) at $450 \mathrm{~nm}$.

Uptake experiment. According to the manufacturer's instructions, NLF-derived exosomes labeled by PKH67 (Sigma-Aldrich, St. Louis, MO, USA) were added into HUVECs. HUVECs were then fixed and processed an immunocytochemical analysis. Photographs were acquired using a TCS SP-5 confocal microscope (Leica Microsystems, Wetzlar, Germany), captured under $400 \mathrm{~Hz}$ with an image resolution of $512 \times 512$ pixels, and then analyzed by Leica Application Suite 2.02.

Western blot analysis. Protein samples were separated using $10 \%$ SDS-PAGE gel and transferred to polyvinylidene fluoride 


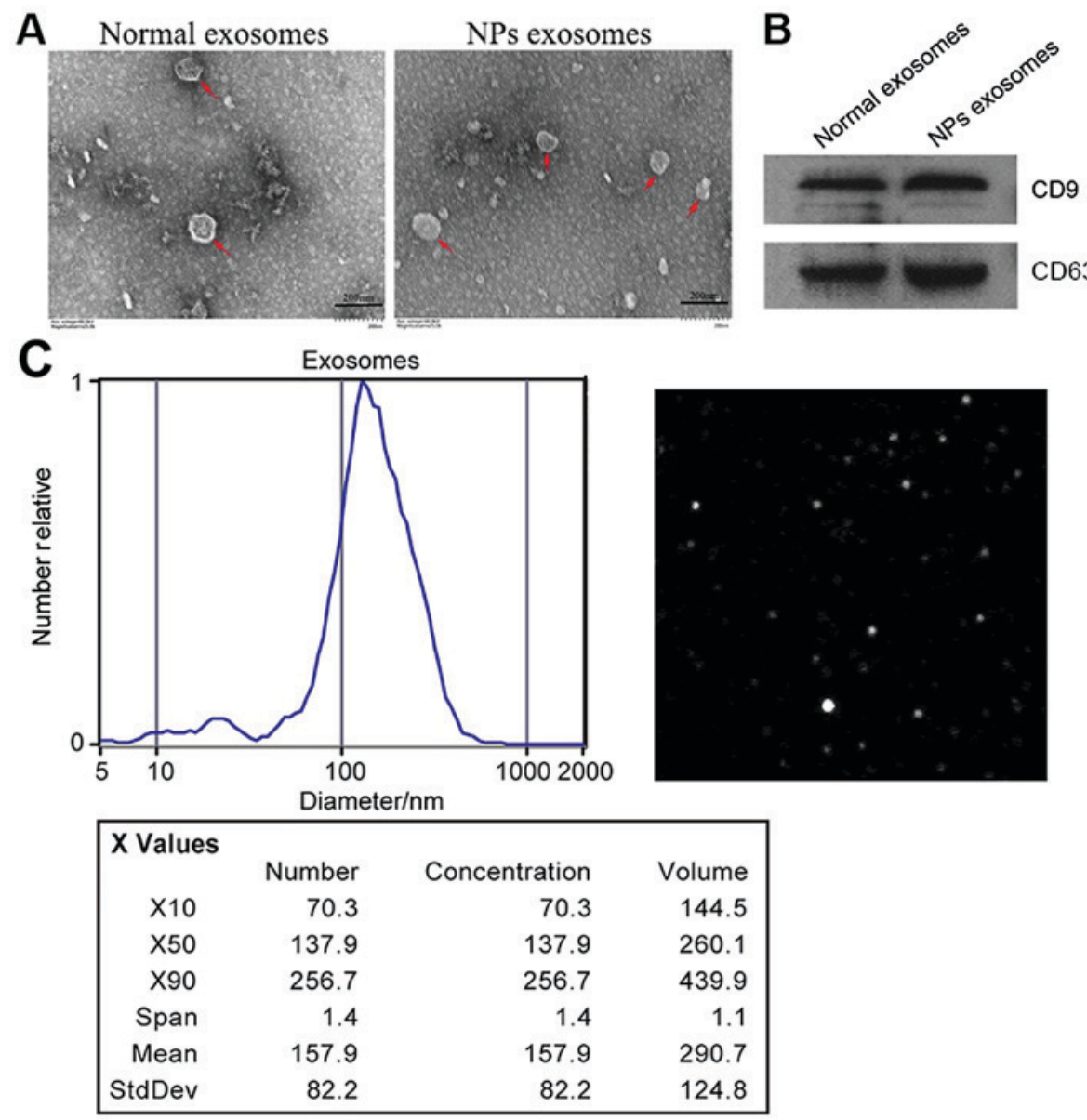

Figure 1. Identification of exosomes from NLF. (A) Representative electron microscopy images of exosomes (indicated by red arrows) purified from NLF (scale bars, $200 \mathrm{~nm}$ ). (B) Western blot analysis of the exosomal markers CD63 and CD9. (C) Nanoparticle Tracking Analysis determined the size of exosomes. NLF, nasal lavage fluid; NP, nasal polyps; CD, cluster of differentiation.

(PVDF) membranes, and then blocked with $5 \%$ non-fat milk. The PVDF membranes were incubated with primary antibody against ADAM10 (1:300; D221496-0100; Sangon Biotech Co., Ltd., Shanghai, China), $\beta$-actin (1:2,000; sc-47778) and CD63 (both from Santa Cruz Biotechnology, Inc., Santa Cruz, CA, USA), CD9 (Abcam, Cambridge, MA, USA), respectively, overnight at $4^{\circ} \mathrm{C}$. Afterwards, the membranes were incubated with horseradish peroxidase-linked immunoglobulin $\mathrm{G}$ (1:5,000; sc-2374; Santa Cruz Biotechnology, Inc.). Gray-scale analysis was performed using Image $\mathrm{J}$ Software to examine the protein bands.

Statistical analysis. Statistical analysis was performed using SPSS 19.0. software (IBM Corp., Armonk, NY, USA). The results were presented as mean \pm standard deviation, and the statistical significance was evaluated using analysis of variance with a Bonferroni post hoc test. P-value $<0.05$ was considered as a statistically significant difference.

\section{Results}

Identification of exosomes in NLF. Based on several reports indicating that exosomes were present in $\operatorname{NLF}(13,14)$, we purified exosomes from the NLF of NPs (NPs exo) and healthy donors (Normal exo). Electron microscopy confirmed the presence of exosomes both in size and morphology (Fig. 1A).
Western blot analysis revealed that the known exosomal markers tetraspanins CD63 and CD9 were highly expressed in NLF-derived exosomes (Fig. 1B). NTA showed vesicles with sizes between 50 and $100 \mathrm{~nm}$ (Fig. 1C).

Influence of NLF-derived exosomes from NPs on angiogenesis and permeability. Since the genesis of NPs was associated with the abnormal angiogenesis and vascular permeability (3), we further evaluated effects of NLF-derived exosomes from NPs on HUVECs. As shown in Fig. 2A, incubation of fluorescent NLF-derived exosomes (green) resulted in transferring of fluorescence to HUVECs. NLF-derived exosomes from NPs were significantly more potent in stimulating tube formation (Fig. 2B), proliferation (Fig. 2C) and permeability (Fig. 2D) of HUVECs.

Expression of ADAM10 in NLF-derived exosomes. Additional, we test to unravel the underlying molecular mechanisms by which NLF-derived exosomes regulate angiogenesis and vascular permeability. Previous studies have found that ADAM10 can influence angiogenesis (20) and vascular permeability (21), and our group had conducted some research on ADAM10 before (22). Based on these data, we detected the expression of ADAM10 in NLF-derived exosomes using western blot. Results showed that ADAM10 was enriched in NLF-derived exosomes from NPs compared to healthy volunteers (Fig. 3). 

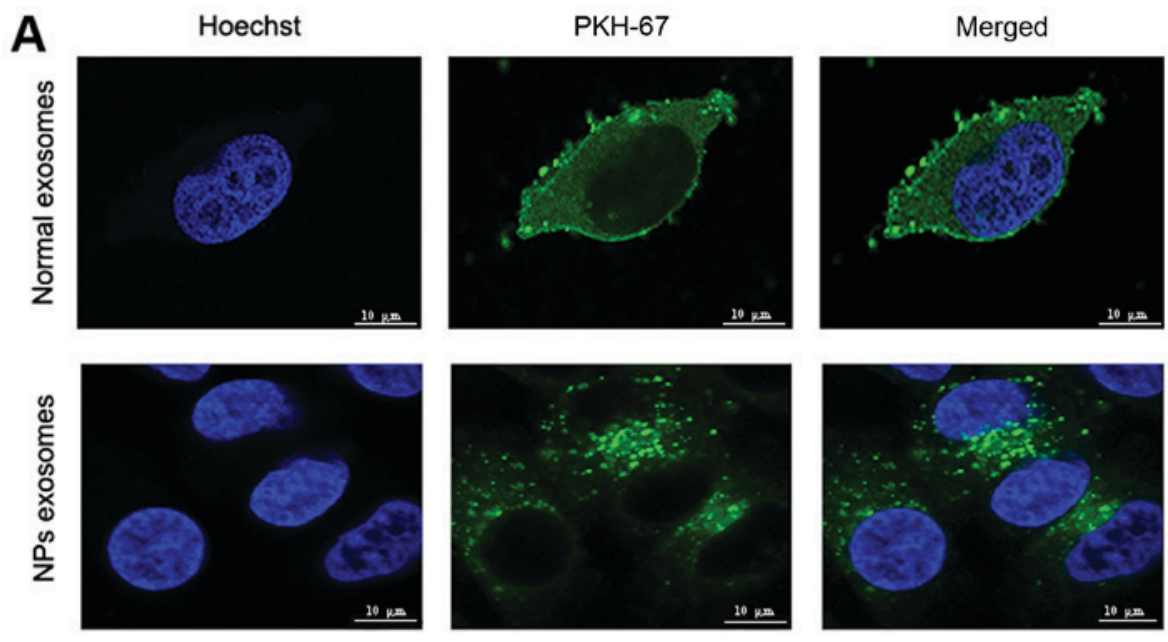

B
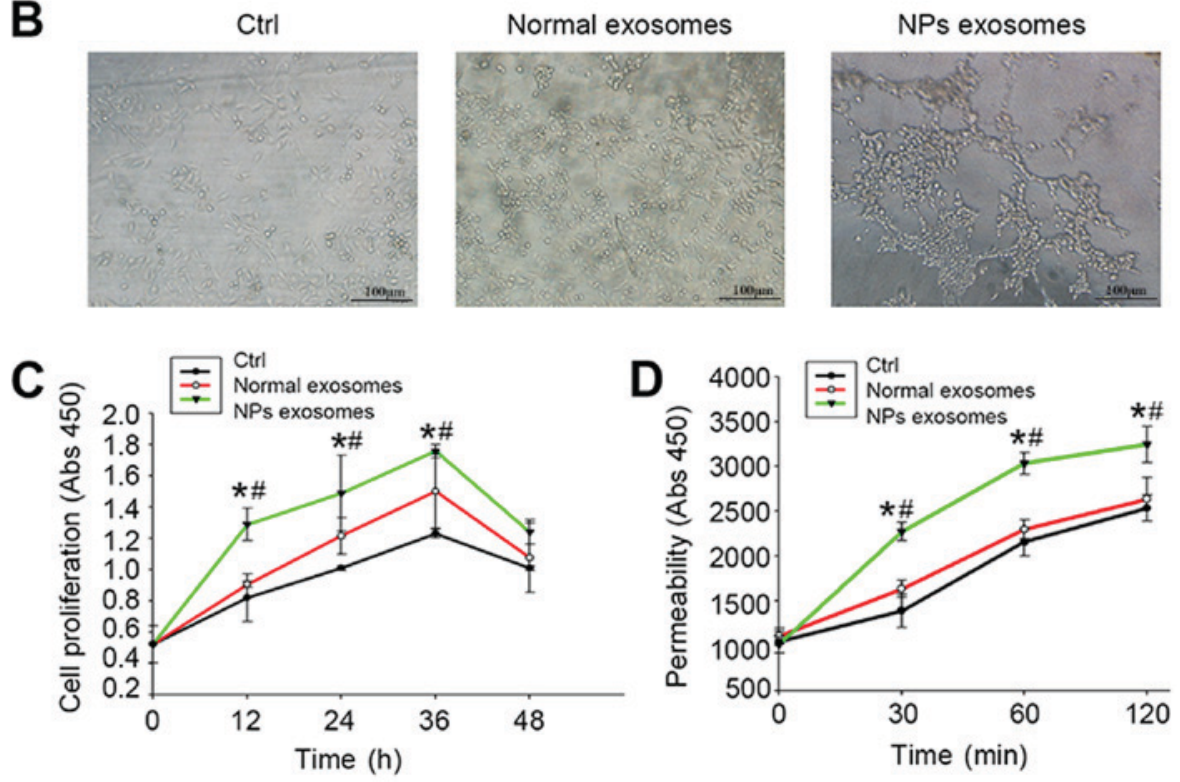

Figure 2. Influence of NLF-derived exosomes from NPs on angiogenesis and permeability of HUVECs. (A) Confocal microscopy analysis of PKH67-labeled (green fluorescence) NLF-derived exosomes uptake by HUVECs following co-culture for $3 \mathrm{~h}$ (scale bars, $10 \mu \mathrm{m}$ ). (B) HUVECs cultured for $24 \mathrm{~h}$ in the absence (Ctrl) or presence of normal and NPs exosomes, and then grown on Matrigel. Pictures are representative photomicrographs from the different groups (scale bars, $10 \mu \mathrm{m}$ ). (C) Effects of NPs exosomes on HUVEC proliferation. (D) Effects of NPs exosomes on HUVEC permeability. All experiments were repeated 3 times and data are presented as the mean \pm standard deviation. ${ }^{*} \mathrm{P}<0.05$ vs. Ctrl; ${ }^{*} \mathrm{P}<0.05$ vs. normal exosomes. NLF, nasal lavage fluid; NP, nasal polyps; HUVECs, human umbilical vein endothelial cells; Ctrl, control.

Above data showed that exosomes existed in NLF. NLF-derived exosomes from NPs promote angiogenesis and vascular permeability. Furthermore, ADAM10 was highly expressed in NLF-derived exosomes from NPs.

\section{Discussion}

NPs are characterized by angiogenesis and interstitial edema with infiltration of inflammatory cells $(30,31)$. As previously described, the blood vessels in NPs were immature and lacking normal innervations. The modifications in these blood vessels suggest angiogenic processes are important for the formation of NPs. And the interstitial edema is probably due to excessive endothelial permeability (32).

To our knowledge, research on exosomes has been expanding in many fields such as cancer $(33,34)$, inflammation (35), immunology (36), diabetes (37) and so on. However, reports on exosomes in NPs are rare. In our study, we found that exosomes exit in NLF collected from NP patients. Isolated exosomes were visualized using transmission electron microscope. Electron micrographs of exosomes from NPs showed both the size and morphology are similar to previous researches $(8,9)$. And then, these exosomes were identified by the western blot, showing the common exosomal surface markers tetraspanins CD9 and CD63. Furthermore, NTA showed vesicles with sizes between 50 and $100 \mathrm{~nm}$. Next, we investigated the influence of NLF-derived exosomes from NPs on HUVECs. Confocal microscopy analysis showed that PKH67-labeled (green) NLF-derived exosomes were uptaken by HUVECs after co-culture for $3 \mathrm{~h}$. However, it would have been more convincing to also use a cell membrane marker to show that there was green fluorescence inside cells. This is a limitation of our study. Furthermore, CCK- 8 assay, in vitro tube formation and permeability assay respectively demonstrated that NPs exo accelerated the proliferation, tube formation and permeability. These results implied that NLF-derived 
A

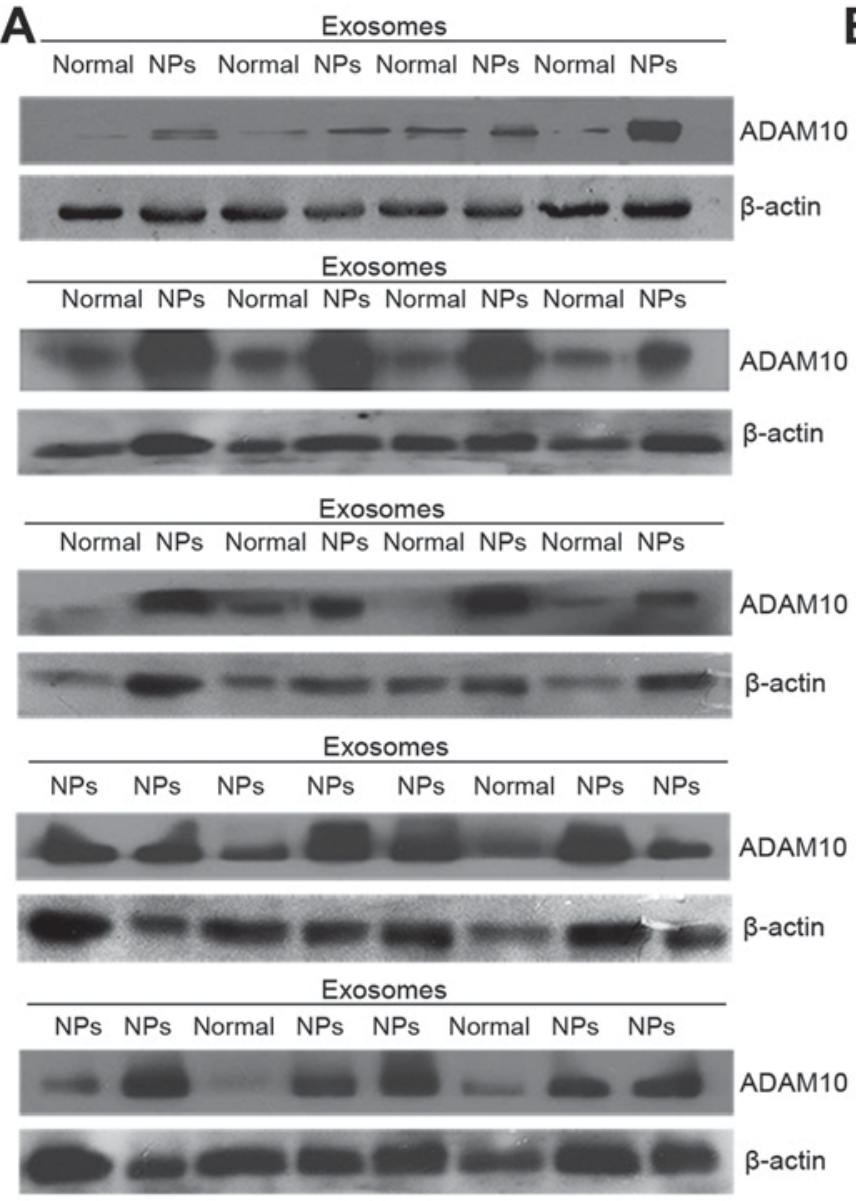

B
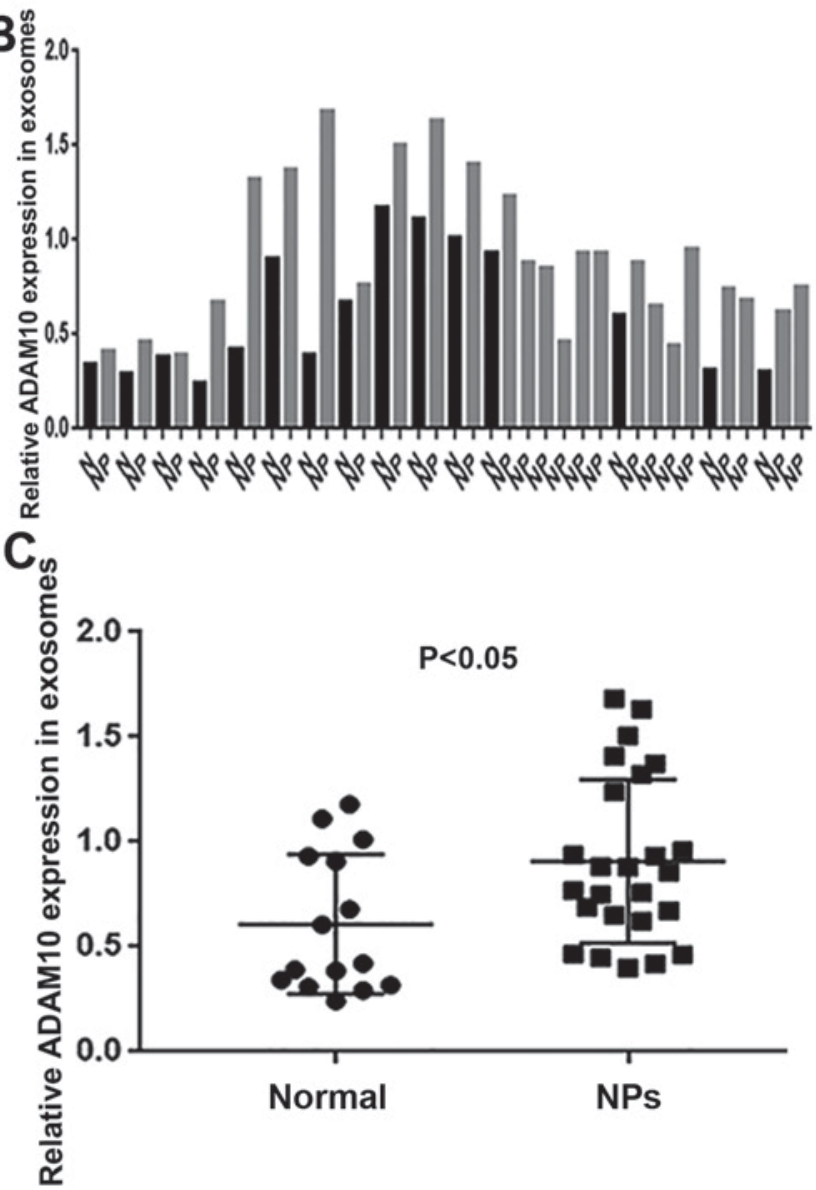

Figure 3. ADAM10 is highly expressed in NLF-derived exosomes from NPs. (A) ADAM10 protein level in exosomes purified from NLF samples from 25 NP patients and 15 healthy volunteers. (B) Grey-scale analysis of all of the ADAM10 protein bands. (C) Comparative analysis of the protein expression levels of ADAM10 in the NPs and normal exosomes. The experiments were carried out in triplicate and data are presented as the mean \pm standard deviation. P $<0.05$, as indicated. ADAM10, A disintegrin and metalloprotease 10; NLF, nasal lavage fluid; NP, nasal polyps.

exosomes from NPs had the function of promoting angiogenesis and vascular permeability. However, exosomes contain abundant protein, mRNA, miRNA and so on $(16,17)$, which bioactive substances are related to the effect of NLF-derived exosomes from NPs on blood vessels? Therefore, we tried to explore the molecular mechanisms underlying the function of NLF-derived exosomes from NPs.

ADAMs are transmembrane proteins carrying an $\mathrm{NH}_{2}$-terminal prodomain, preceding a metalloproteinase (catalytic) domain, which is followed by a disintegrin, a cysteine-rich, a transmembrane, and finally a cytoplasmic domain (38). Previous researches have shown that ADAMs are active in NPs. An immunohistochemical study has found increased expression of ADAM33 protein in vessels and stroma of NPs tissues (39). Expression of ADAM8 was higher in NPs tissues than in normal nasal mucosa, and there was a positive correlation between the strength of ADAM8 immunostaining and the level of inflammation in NPs tissues (40).

ADAM10 is a ubiquitous transmembrane metalloprotease belonging to ADAM-family. It has been shown that ADAM10 is associated with angiogenesis (20) and vascular permeability (21), and ADAM10 has been found in healthy nasal exosomes recently (15). However, the expression level of ADAM10 in NLF-derived exosomes from NPs has not been demonstrated. In this study, we detected ADAM10 expression in exosomes using western blot, showing that ADAM10 was overexpressed in NLF-derived exosomes from NPs compared to the healthy volunteers. Accordingly, we speculated that the role of NLF-derived exosomes from NPs in promoting angiogenesis and vascular permeability may be associated with ADAM10. However, this is just our initial speculation. In the future, we plan to do some other experiments such as inhibition of ADAM10 in the NLF both in vivo (zebrafish model) and in vitro (human nasal epithelial cells, hNECs) to validate our hypothesis. Besides, ADAM10 is known associated with cell member. As for which cells did release the exosomes with ADAM10 has not been known. Further reseach on exosomes isolated from hNECs may answer the question.

In conclusion, this study shows for the first time that, NLF-derived exosomes from NPs promote angiogenesis and vascular permeability and contain abundant ADAM10. Further research on the effects of ADAM10-containing exosomes derived from NLF on angiogenesis and vascular permeability may reveal the underlying molecular mechanisms of NPs. Even though the presence of exosomes in human NLF may be useful as a diagnostic indicator of upper respiratory tract disease, it remains unclear whether these extracellular components are causative. In the future, we plan to do research on the correlation between NLF-derived exosomes and severity of disease, which may help answering this question. 


\section{Acknowledgements}

The authors would like to thank Professor Yiwen You (Department of Otorhinolaryngology, Affiliated Hospital of Nantong University, Jiangsu, China) for her general support and assistance.

\section{Funding}

This study was supported by grants from Jiangsu Provincial Commission of Health and Family Planning (grant no. H201523) and Nantong Clinical Research Project (grant no. HS2016001).

\section{Availability of data and materials}

All data analysed during the present study are included in this published article, and the datasets are available from the corresponding author on reasonable request.

\section{Authors' contributions}

JC and HY made substantial contributions to conception and design, and gave final approval to the manuscript to be published. HN and BY recruited the appropriate patients, and collected nasal lavage fluid from them. YS, LB, DW and TZ conducted the experiments and acquired the data. WZ and JZ analyzed and interpreted data, and were the major contributors in writing the manuscript. LC provided theoretical and technical support, and revised the manuscript critically for important intellectual content. All authors read and approved the final manuscript.

\section{Ethics approval and consent to participate}

Written informed consent was obtained from all patients and healthy volunteers, and the study was approved by the Medical Ethics Committee of the Affiliated Hospital of Nantong University (Jiangsu, China; ethical review no. 2017-L088).

\section{Consent for publication}

Written informed consent was obtained from all patients and healthy volunteers.

\section{Competing interests}

The authors declare that they have no competing interests.

\section{References}

1. Fokkens WJ, Lund VJ, Mullol J, Bachert C, Alobid I, Baroody F, Cohen N, Cervin A, Douglas R, Gevaert P, et al: EPOS 2012: European position paper on rhinosinusitis and nasal polyps 2012. A summary for otorhinolaryngologists. Rhinology 50: 1-12, 2012.

2. Wang C, Lou H, Wang X, Wang Y, Fan E, Li Y, Wang H, Bachert $\mathrm{C}$ and Zhang L: Effect of budesonide transnasal nebulization in patients with eosinophilic chronic rhinosinusitis with nasal polyps. J Allergy Clin Immunol 135: 922-929.e6, 2015.

3. Coste A, Rateau JG, Roudot-Thoraval F, Chapelin C, Gilain L, Poron F, Peynegre R, Bernaudin JF and Escudier E: Increased epithelial cell proliferation in nasal polyps. Arch Otolaryngol Head Neck Surg 122: 432-436, 1996.
4. Yukitatsu Y, Hata M, Yamanegi K, Yamada N, Ohyama H, Nakasho K, Kojima Y, Oka H, Tsuzuki K, Sakagami M and Terada N: Decreased expression of VE-cadherin and claudin-5 and increased phosphorylation of VE-cadherin in vascular endothelium in nasal polyps. Cell Tissue Res 352: 647-657, 2013.

5. Gosepath J, Brieger J, Lehr HA and Mann WJ: Expression, localization, and significance of vascular permeability/vascular endothelial growth factor in nasal polyps. Am J Rhinol 19: 7-13, 2005.

6. Record M, Subra C, Silvente-Poirot S and Poirot M: Exosomes as intercellular signalosomes and pharmacological effectors. Biochem Pharmacol 81: 1171-1182, 2011.

7. Kulshreshtha A, Ahmad T, Agrawal A and Ghosh B: Proinflammatory role of epithelial cell-derived exosomes in allergic airway inflammation. J Allergy Clin Immunol 131: 1194-1203, 1203. e1-e14, 2013.

8. Honegger A, Schilling D, Bastian S, Sponagel J, Kuryshev V, Sültmann H, Scheffner M, Hoppe-Seyler K and Hoppe-Seyler F: Dependence of intracellular and exosomal microRNAs on viral E6/E7 oncogene expression in HPV-positive tumor cells. PLoS Pathog 11: e1004712, 2015.

9. You B, Cao X, Shao X, Ni H, Shi S, Shan Y, Gu Z and You Y: Clinical and biological significance of HAX-1 overexpression in nasopharyngeal carcinoma. Oncotarget 7: 12505-12524, 2016.

10. You Y, Shan Y, Chen J, Yue H, You B, Shi S, Li X and Cao X: Matrix metalloproteinase 13-containing exosomes promote nasopharyngeal carcinoma metastasis. Cancer Sci 106: 1669-1677, 2015.

11. Nakayama A: Proteomic analysis of urinary exosomes. Rinsho Byori 62: 684-691, 2014 (In Japanese).

12. Näslund TI, Paquin-Proulx D, Paredes PT, Vallhov H, Sandberg JK and Gabrielsson S: Exosomes from breast milk inhibit HIV-1 infection of dendritic cells and subsequent viral transfer to $\mathrm{CD}^{+} \mathrm{T}$ cells. AIDS 28: 171-180, 2014.

13. Lässer C, O'Neil SE, Ekerljung L, Ekström K, Sjöstrand M and Lötvall J: RNA-containing exosomes in human nasal secretions. Am J Rhinol Allergy 25: 89-93, 2011.

14. Choi EB, Hong SW, Kim DK, Jeon SG, Kim KR, Cho SH, Gho YS, Jee YK and Kim YK: Decreased diversity of nasal microbiota and their secreted extracellular vesicles in patients with chronic rhinosinusitis based on a metagenomic analysis. Allergy 69: 517-526, 2014.

15. Lässer C, O'Neil SE, Shelke GV, Sihlbom C, Hansson SF, Gho YS, Lundbäck B and Lötvall J: Exosomes in the nose induce immune cell trafficking and harbour an altered protein cargo in chronic airway inflammation. J Transl Med 14: 181, 2016.

16. Sampey GC, Saifuddin M, Schwab A, Barclay R, Punya S, Chung MC, Hakami RM, Zadeh MA, Lepene B, Klase ZA, et al: Exosomes from HIV-1-infected cells stimulate production of pro-inflammatory cytokines through trans-activating response (TAR) RNA. J Biol Chem 291: 1251-1266, 2016.

17. Wang X, Gu H, Qin D, Yang L, Huang W, Essandoh K, Wang Y, Caldwell CC, Peng T, Zingarelli B and Fan GC: Exosomal miR-223 contributes to mesenchymal stem cell-elicited cardioprotection in polymicrobial sepsis. Sci Rep 5: 13721, 2015.

18. Lee SL, Rouhi P, Dahl Jensen L, Zhang D, Ji H, Hauptmann G, Ingham $\mathrm{P}$ and Cao Y: Hypoxia-induced pathological angiogenesis mediates tumor cell dissemination, invasion, and metastasis in a zebrafish tumor model. Proc Natl Acad Sci USA 106: 19485-19490, 2009.

19. Kalani A, Kamat PK, Chaturvedi P, Tyagi SC and Tyagi N: Curcumin-primed exosomes mitigate endothelial cell dysfunction during hyperhomocysteinemia. Life Sci 107: 1-7, 2014.

20. Isozaki T, Rabquer BJ, Ruth JH, Haines GK III and Koch AE: ADAM-10 is overexpressed in rheumatoid arthritis synovial tissue and mediates angiogenesis. Arthritis Rheum 65: 98-108, 2013.

21. Blobel CP: Metalloprotease-disintegrins: Links to cell adhesion and cleavage of TNF alpha and Notch. Cell 90: 589-592, 1997.

22. You B, Shan Y, Shi S, Li X and You Y: Effects of ADAM10 upregulation on progression, migration and prognosis of nasopharyngeal carcinoma. Cancer Sci 106: 1506-1514, 2015.

23. Chueh HW, Park SK, Hur DY and Bae WY: Expression profile of ADAM10 and ADAM17 in allergic rhinitis. Int Forum Allergy Rhinol 5: 1036-1041, 2015.

24. Schulz B, Pruessmeyer J, Maretzky T, Ludwig A, Blobel CP, Saftig P and Reiss K: ADAM10 regulates endothelial permeability and T-cell transmigration by proteolysis of vascular endothelial cadherin. Circ Res 102: 1192-1201, 2008. 
25. Donners MM,Wolfs IM,Olieslagers S, Mohammadi-MotahhariZ, Tchaikovski V, Heeneman S, van Buul JD, Caolo V, Molin DG, Post MJ and Waltenberger J: A disintegrin and metalloprotease 10 is a novel mediator of vascular endothelial growth factor-induced endothelial cell function in angiogenesis and is associated with atherosclerosis. Arterioscler Thromb Vasc Biol 30: 2188-2195, 2010.

26. Reiss K, Cornelsen I, Husmann M, Gimpl G and Bhakdi S: Unsaturated fatty acids drive disintegrin and metalloproteinase (ADAM)-dependent cell adhesion, proliferation, and migration by modulating membrane fluidity. J Biol Chem 286: 26931-26942, 2011.

27. Howarth PH, Persson CG, Meltzer EO, Jacobson MR, Durham SR and Silkoff PE: Objective monitoring of nasal airway inflammation in rhinitis. J Allergy Clin Immunol 115 (3 Suppl 1): S414-S441, 2005.

28. Valadi H,Ekström K, Bossios A, Sjöstrand M,Lee JJ and Lötvall JO Exosome-mediated transfer of mRNAs and microRNAs is a novel mechanism of genetic exchange between cells. Nat Cell Biol 9: 654-659, 2007

29. Romancino DP, Paterniti G, Campos Y, De Luca A, Di Felice V, d'Azzo A and Bongiovanni A: Identification and characterization of the nano-sized vesicles released by muscle cells. FEBS Lett 587: 1379-1384, 2013.

30. Hellquist HB: Nasal polyps update. Histopathology. Allergy Asthma Proc 17: 237-242, 1996.

31. Slavin RG: Nasal polyps and sinusitis. Clin Allergy Immunol 16 295-309, 2002

32. Watanabe K and Komatsuzaki A: Ultrastructural findings of capillaries in nasal polyps. Rhinology 30: 49-56, 1992.

33. Higginbotham JN, Demory BM, Gephart JD, Franklin JL, Bogatcheva G, Kremers GJ, Piston DW, Ayers GD, McConnell RE, Tyska MJ and Coffey RJ: Amphiregulin exosomes increase cancer cell invasion. Curr Biol 21: 779-786, 2011.
34. Hood JL, San RS and Wickline SA: Exosomes released by melanoma cells prepare sentinel lymph nodes for tumor metastasis. Cancer Res 71: 3792-3801, 2011.

35. Martinez-Bravo MJ, Wahlund CJ, Qazi KR, Moulder R, Lukic A, Rådmark O, Lahesmaa R, Grunewald J, Eklund A and Gabrielsson S: Pulmonary sarcoidosis is associated with exosomal vitamin D-binding protein and inflammatory molecules. J Allergy Clin Immunol 139: 1186-1194, 2016.

36. Zhu M, Li Y, Shi J, Feng W, Nie G and Zhao Y: Exosomes as extrapulmonary signaling conveyors for nanoparticle-induced systemic immune activation. Small 8: 404-412, 2012.

37. Bashratyan R, Sheng H, Regn D, Rahman MJ and Dai YD: Insulinoma-released exosomes activate autoreactive marginal zone-like B cells that expand endogenously in prediabetic NOD mice. Eur J Immunol 43: 2588-2597, 2013.

38. Dreymueller D, Uhlig S and Ludwig A: ADAM-family metalloproteinases in lung inflammation: Potential therapeutic targets. Am J Physiol Lung Cell Mol Physiol 308: L325-L343, 2015.

39. Erbek SS, Erinanc H, Erbek S, Topal O and Kiyici H: Expression of a disintegrin and metalloproteinase 33 protein in nasal polyposis: An immunohistochemical study. Am J Rhinol Allergy 24: 79-82, 2010.

40. Erbek SS, Hizal E, Erinanc $\mathrm{H}$ and Erbek S: Expression of a disintegrin and metalloproteinase 8 by inflammatory cells in nasal polyps. Am J Rhinol Allergy 27: 151, 2013.

This work is licensed under a Creative Commons Attribution-NonCommercial-NoDerivatives 4.0 International (CC BY-NC-ND 4.0) License. 